\title{
Nutzen und Grenzen von Modellökosystemen zur Erfassung der Wirkung von Schadstoffen auf benthische Lebensgemeinschaften
}

\author{
Marvin Brinke • Sebastian Höss · Walter Traunspurger · Peter Heininger
}

Eingegangen: 11. März 2009/Akzeptiert: 29. April 2009/Online veröffentlicht: 14. Mai 2009

(C) Springer-Verlag 2009

\begin{abstract}
Zusammenfassung Ziel dieses Kurzbeitrages ist es, einen Einblick in den Nutzen und die Grenzen von Modellökosystemen bei der Untersuchung von Schadstoffeffekten auf benthische Lebensgemeinschaften, insbesondere der Meiofauna, zu geben. Eine Vielzahl von Schadstoffen akkumulieren in Sedimenten, die somit eine komplexe Belastung für benthische Organismen darstellen. Neben direkten Effekten spielen indirekte Effekte eine große Rolle. Durch veränderte Konkurrenz oder Räuber-Beute-Beziehungen können vielfältige Effekte im benthischen Nahrungsnetz ausgelöst werden, die nicht durch eine direkte Toxizität vorhandener Schadstoffe erklärbar sind. Es werden daher sowohl direkte als auch indirekte Effekte aus zwei Mikrokosmosstudien mit dem Schwermetall Cadmium und dem Veterinärpharmazeutikum Ivermectin vorgestellt. In beiden Untersuchungen kam es zu klaren Veränderungen, sowohl der Abundanz als auch der Zusammensetzung der Lebensgemeinschaft der Meiofauna. Letztlich sind die Ziele von Studien mit Modellökosystemen, Effekte eines bestimmten Schadstoffs auf natürliche Lebensgemeinschaften im Freiland vorauszusagen sowie Ergebnisse von Freilandstudien erklären zu können. Die beiden durchgeführten Studien zeigten, dass die Untersuchung
\end{abstract}

Verantwortliche Herausgeber: Jan Schwarzbauer · Peter Heininger · Evelyn Claus

Marvin Brinke $(\varangle) \cdot$ Walter Traunspurger

Tierökologie, Universität Bielefeld,

Morgenbreede 45, 33615 Bielefeld, Deutschland

E-Mail: marvin.brinke@uni-bielefeld.de

Sebastian Höss

ECOSSA,

Giselastr. 6, 82319 Starnberg, Deutschland

Peter Heininger

Bundesanstalt für Gewässerkunde,

Am Mainzer Tor 1, 56068 Koblenz, Deutschland der Meiofauna in Mikrokosmen zur Erreichung dieser Ziele wesentlich beitragen kann. Darüber hinaus können Mikrokosmen mit Meiofauna möglicherweise einen Beitrag zur Risikobewertung leisten, indem sie die effektgeleitete Analyse (EDA) bei der Identifizierung von effektverursachenden Schadstoffen in komplexen Umweltproben unterstützen.

Schlüsselwörter Modellökosysteme · Mikrokosmen · Meiofauna $\cdot$ Lebensgemeinschaft $\cdot$ Nematoden $\cdot$ Sediment

Assessment of the effects caused by contaminants on meiobenthic communities: prospects and constraints of freshwater model ecosystems

Abstract The aim of this short article is to deliver insight into the prospects and constraints of model ecosystems in the assessment of effects of contaminants on benthic communities, especially the meiofauna. Numerous substances accumulate in sediments and thus a complex contamination exists which is able to impair benthic organisms. Besides direct effects, indirect effects are of importance and should be considered. Changes in competition and predator-prey-relationships lead to a variety of effects in the benthic food web that cannot be explained by direct toxicity of contaminants. In this article two microcosm studies, one with the heavy metal cadmium and one with the veterinary pharmaceutical Ivermectin, are presented which both showed direct as well as indirect effects. A significant change in abundance and composition of the meiofauna community has been observed. The prediction of field-effects of a certain substance as well as the interpretation of field data are the aims of model ecosystems. Both studies proofed that the investigation of meiofauna in microcosms is a promising tool to achieve these aims. Furthermore, microcosms with meiofauna might 
be able to assist the effect-directed analysis (EDA) in a risk assessment by identifying the effect-causing contaminants of complex environmental samples.

Keywords Model ecosystems $\cdot$ Microcosms $\cdot$ Meiofauna Community $\cdot$ Nematodes $\cdot$ Sediment

\section{Einleitung}

Durch Schadstoffeinträge in Gewässer werden neben dem Wasserkörper auch die Sedimente belastet. Häufig kommt es durch die Bindung der Schadstoffe an die Sedimentpartikel zu einer Anreicherung (Bryan und Langston 1992). Dies führt nicht nur zu hohen Konzentrationen einzelner Schadstoffe, sondern auch zur Akkumulation einer Vielzahl von Schadstoffen in den Sedimenten. Somit sind benthische Organismen und Lebensgemeinschaften oft einer hohen und komplexen Schadstoffbelastung ausgesetzt. Vor allem die Meiofauna (z. B. Nematoden, Anneliden, Tardigraden, Rotatorien, Ostracoden), der arten- und individuenreichste Teil der benthischen Lebensgemeinschaft (Giere 2009), ist davon betroffen, da diese Taxa ihren kompletten Lebenszyklus innerhalb des Sediments verbringen und so in allen Lebensphasen gegenüber den Schadstoffen exponiert sind (Coull und Chandler 1992). Allerdings ist es nicht einfach, klare Ursache-Wirkungsbeziehungen zwischen Belastungsmustern und beobachteten Veränderungen in benthischen Lebensgemeinschaften herzustellen. Es ist selten möglich, den effektverursachenden Schadstoff eindeutig zu identifizieren. Ein Zusammenspiel aus Exposition, Bioverfügbarkeit und Empfindlichkeit entscheidet, ob Schadstoffe eine Wirkung hervorrufen können oder nicht. Dabei ist zu beachten, dass eine beobachtete Veränderung der Lebensgemeinschaft auch indirekt über das Nahrungsnetz und nicht nur durch einen direkten Effekt eines Schadstoffs erfolgen kann. Dies ist zum Beispiel der Fall, wenn ein Schadstoff eine Wirkung auf Konkurrenten oder Räuber hat und die biotischen Bedingungen so verändert werden, dass sich dies in der Struktur der Lebensgemeinschaft niederschlägt. Solche indirekten Effekte können nur aufgedeckt werden, wenn die Lebensgemeinschaft und somit das benthische Nahrungsnetz als Ganzes betrachtet wird. Modellökosysteme bieten eine gute Möglichkeit, direkte und indirekte Schadstoffeffekte auf Lebensgemeinschaften unter kontrollierten Bedingungen zu untersuchen. Die Meiofauna zeigt Eigenschaften, die sie für Studien in Modellökosystemen prädestiniert: 1) geringe Größe (meist 0,5-5 mm), 2) hohe Abundanz (1-10 Millionen Individuen pro Quadratmeter), 3) kurze Generationszeiten (Wochen bis Monate), 4) viele Ernährungstypen (Bakterien-, Algen-, Pilz-, Pflanzen-, Allesfresser, Räuber). Diese Eigenschaften erlauben ökologisch relevante Modellökosysteme von geringer Größe und kurzer Laufzeit bei gleichzeitig hoher statistischer Aussagekraft. Zudem nimmt die Meiofauna durch ihre hohe Abundanz und Artenvielfalt eine Schlüsselposition im Nahrungsnetz zwischen der Mikrofauna (z. B. Bakterien, Pilze, Protozoen) und der Makrofauna (z.B. Schnecken, Muscheln, Insektenlarven) ein. Veränderungen im Nahrungsangebot (z.B. Bakterien, Algen) und das Fehlen von potenziellen Räubern wirkt sich direkt auf die Meiofauna aus. Es ist überraschend, dass die Erfassung der Meiofauna in Modellökosystemen zur Beantwortung ökotoxikologischer Fragestellungen im Vergleich zur Makrofauna meist vernachlässigt wurde (Kennedy und Jacoby 1999). Bisher wurde vorwiegend im marinen bzw. ästuaren Lebensraum der Effekt von Schadstoffen auf die Meiofauna in Modellökosystemen untersucht (z.B. Austen und McEvoy 1997; Austen und Somerfield 1997; Beyrem et al. 2007; Gyedu-Ababio und Baird 2006; Millward et al. 2001; Schratzberger et al. 2002; Suderman und Thistle 2003). Ebenso wurden in terrestrischen Modellökosystemen insbesondere die Nematoden als Bioindikatoren verwendet (z. B. Korthals et al. 1996; Smit et al. 2002). Hingegen wurde für Süßgewässer die Meiofauna bisher nur sehr selten in Modellökosystemen berücksichtigt (z. B. Höss et al. 2004). Daher sind weitere Studien mit Modellökosystemen notwendig, um die Wirkung von Schadstoffen auf das Meiobenthos in Seen und Fließgewässern besser zu verstehen.

\section{Ergebnisse und Diskussion}

In zwei Mikrokosmosstudien mit einem Schwermetall (Cadmium; Cd) und einem Veterinärpharmazeutikum (Ivermectin; IVM) sollte zum einen der Effekt der Schadstoffe auf die limnische Meiofauna untersucht werden, um sowohl Daten aus Freilandstudien besser interpretieren, als auch Voraussagen über die zu erwartende Wirkung im Freiland machen zu können. Zum anderen sollte die Eignung der Meiofauna für Higher-Tier-Studien in Modellökosystemen überprüft werden (Publikationen in Vorbereitung). Der Eintrag von $\mathrm{Cd}\left(10,100\right.$ und $\left.1000 \mathrm{mg} \mathrm{kg}^{-1} \mathrm{TG}\right)$ in das Sediment führte zu Veränderungen der Lebensgemeinschaften der Meiofauna als Ganzes und der Nematoden im Speziellen. Bei diesen hohen nominellen Sedimentkonzentrationen ist aber zu berücksichtigen, dass die höchste je im Versuchsverlauf gemessene Cd-Konzentration im Porenwasser nur $232 \mu \mathrm{g} \mathrm{L}^{-1}$ betrug. Am deutlichsten waren die Veränderungen bei der höchsten Schwermetallkonzentration. Hier profitierten räuberische Nematoden, die bisher als eher schadstoffsensitiv galten, von der Cd-Belastung, während die eher schadstofftoleranten, bakterienfressenden Nematoden in ihrer Häufigkeit deutlich abnahmen. Dieses Ergebnis bestätigt ein Phänomen, das bereits in einer Freilanduntersuchung von Fließgewässern beobachtet wurde (Heininger et al. 2007), steht aber im Widerspruch zu bisherigen Beobachtungen 
für terrestrische und marine Nematoden (Bongers und Ferris 1999; Bongers et al. 2001). Die Veränderungen der Nematodenlebensgemeinschaft könnten durch Effekte auf konkurrierende, makrobenthische Räuber indirekt induziert worden sein. Eine andere Erklärung könnten unterschiedliche Cd-Expositionen für die verschiedenen Ernährungstypen der Nematoden (Bakterienfresser, Räuber usw.) und daraus resultierende Unterschiede in der Bioverfügbarkeit von $\mathrm{Cd}$ sein. In den niedrigen Cd-Konzentrationen (10 und $100 \mathrm{mg} \mathrm{kg}^{-1} \mathrm{TG}$ ) dominierten neben den Nematoden auch die Anneliden, während in der höchsten Konzentration die Anneliden deutlich abnahmen. Darüber hinaus konnten bereits bei niedrigen Cd-Konzentrationen schon erste Veränderungen der Nematodenlebensgemeinschaft auf Artniveau beobachtet werden. Auch das Pharmazeutikum Ivermectin $\left(0,6,6,2\right.$ und $\left.31 \mu \mathrm{g} \mathrm{kg}^{-1} \mathrm{TG}\right)$ verursachte in Mikrokosmen eine deutliche Veränderung der Lebensgemeinschaft. Als besonders empfindlich erwiesen sich die benthischen Microcrustaceen (Cladoceren, Ostracoden, Copepoden) und die Nematoden. Die bereits in Biotests ermittelte hohe Empfindlichkeit von Daphnien, als Vertreter der Crustaceen (Garric et al. 2007; Halley et al. 1989), konnte somit auch für natürliche Populationen von benthischen Organismengruppen der Crustaceen gezeigt werden. Die Studie zeigte weiterhin, dass Ivermectin, das als Antiparasitikum gegen parasitische Nematoden eingesetzt wird, auch einen deutlichen Effekt auf Abundanz und Diversität freilebender Nematoden hat. Ähnlich wie in den Modellökosystemen mit Cadmium hatten räuberische Nematoden bei der höchsten Ivermectinbelastung ( $\left.31 \mu \mathrm{g} \mathrm{kg}^{-1} \mathrm{TG}\right)$ einen sehr hohen Anteil. Dies ist ein weiterer Hinweis, dass die Arten und Ernährungstypen der Süßwassernematoden anders auf Schadstoffbelastungen reagieren als terrestrische und marine Nematodenarten. Ein positiver Schadstoffeffekt des Ivermectins wurde ebenfalls beobachtet. Tardigraden zeigten eine Abundanzzunahme mit steigender Ivermectinkonzentration, die vermutlich auf indirekte Effekte durch fehlende Konkurrenten zurückzuführen ist. Diese ersten Studien zeigen, dass Untersuchungen der Meiofauna, insbesondere der Nematoden, in Modellökosystemen geeignet sind, Effekte von Schadstoffen auf benthische Süßwasserlebensgemeinschaften zu erfassen. Besonders bei höheren Schadstoffkonzentrationen sind immer noch meiobenthische Organismen in genügender Anzahl vorhanden und können so als Bioindikatoren dienen. Die Studien geben zudem einen Einblick, ob Änderungen der Zusammensetzung auf einem direkten Effekt des Schadstoffes beruhen oder ob indirekte Effekte vorliegen, die wesentlich zur Veränderung der Lebensgemeinschaft beitragen können. Auch wenn in Modellökosystemen meist keine eindeutige Trennung von direkten und indirekten Effekten möglich ist, da sich diese überlagern und nicht immer alle Komponenten des benthischen Nahrungsnetzes erfasst werden (z.B. Abundanz und Arten der
Bakterien und Protozoen), sind Studien mit Modellökosystemen ein erster wichtiger Schritt, um beobachtete Veränderungen im Freiland zu erklären. Im Zusammenspiel mit anderen ökotoxikologischen Methoden, wie z.B. Toxizitätstests, Artenempfindlichkeitsverteilungen (SSD) und effektgeleiteter Analytik (EDA), können Wirkungsursachen erkannt und im Sinne eines integrierten Gewässerschutzes beseitigt werden.

\section{Ausblick}

Welche Rolle können Modellökosysteme in einer Risikobewertung haben? Das zur Bewertung von belasteten Sedimenten von Long und Chapman (1985) vorgeschlagene Konzept der Sedimentqualitätstriade (SQT) beinhaltet drei wesentliche Bestandteile: 1) Sedimentanalytik zur Bestimmung von Schadstoffkonzentrationen, 2) Ermittlung der Toxizität der Sedimente durch Biotests und 3) Untersuchung des Effekts der belasteten Sedimente auf die natürliche in situ Lebensgemeinschaft. Eine Ergänzung bzw. Erweiterung dieses Konzepts ist nicht nur sinnvoll, sondern auch wünschenswert (Chapman und Hollert 2006). Die Anwendung von Modellökosystemen bildet ein Bindeglied zwischen der Schadstoffanalytik und der Analyse der in situ Lebensgemeinschaften und ist damit eine sinnvolle Ergänzung zur Triade (im Sinne des Weight-of-Evidence-Ansatzes; Abb. 1). Durch die Verknüpfung mit anderen, die Triade ergänzenden, Methoden, könnten allerdings noch wertvolle Zusatzinformationen gewonnen werden. Die effektgeleitete Analytik (EDA) bietet die Möglichkeit, durch Fraktionie-

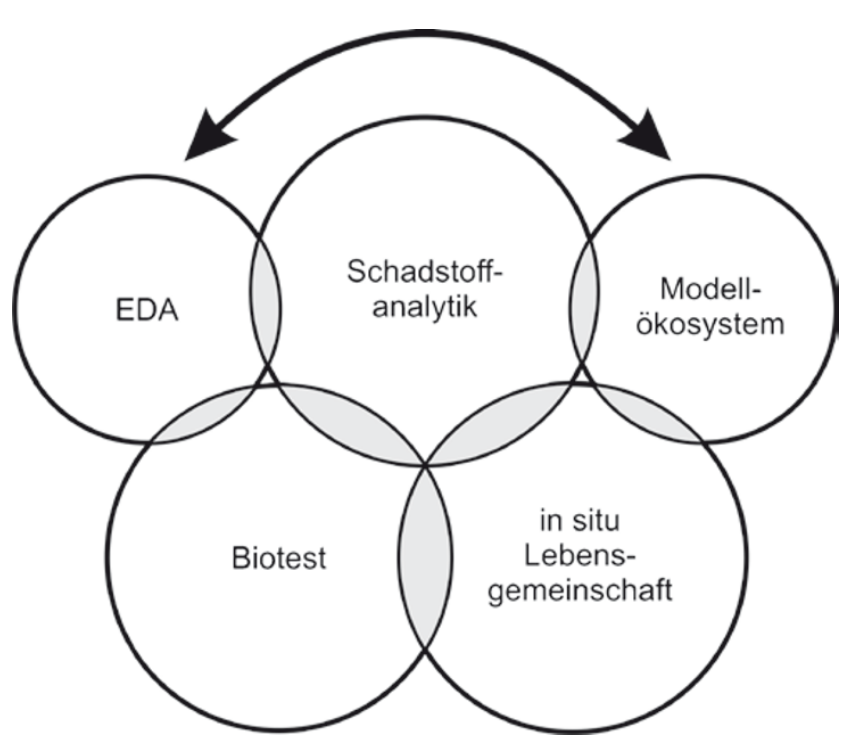

Abb. 1 Eine mit den Beweislinien effektgeleitete Analytik (EDA) und Modellökosystem (z. B. Mikrokosmen) erweiterte Sedimentqualitätstriade (in Anlehnung an Chapman und Hollert 2006) 
rung von Umweltproben und Testung dieser Fraktionen mittels Biotests den effektverursachenden Schadstoff $\mathrm{zu}$ identifizieren (Brack 2003). Eine Schwachstelle der EDA ist allerdings die ökologische Relevanz, da eine realistische Exposition und Bioverfügbarkeit, die vergleichbar zum natürlichen Sediment ist, meist nicht berücksichtigt wird. Ein möglicher Ansatz könnte daher die Kombination aus EDA und Mikrokosmen sein (siehe Abb. 1). Diese Mikrokosmen sollten unbelastetes Sediment mit einer natürlichen Lebensgemeinschaft enthalten. Indem man die Mikrokosmen mit einer, im Rahmen einer EDA, als toxisch identifizierte Fraktion belastet, kann überprüft werden, ob diese Fraktion auch tatsächlich die im Freiland gefundenen Effekte verursacht. Umgekehrt könnten fälschlicherweise durch Biotests als nicht toxisch klassifizierte Fraktionen im Mikrokosmos doch einen Effekt auf die Lebensgemeinschaft und Artenzusammensetzung ausgewählter Organismengruppen auslösen. Wenn es gelingt, ausreichende Mengen der entsprechenden Fraktionen aus den Umweltproben zu gewinnen, dann könnten Mikrokosmen mit der Meiofauna aufgrund ihrer Eigenschaften (geringe Größe der Mikrokosmen, Laufzeit ab 1-2 Monate, hohe Individuen- und Artenzahlen, hohe statistische Aussagekraft) solche Studien realisierbar machen und einen weiteren Beitrag für künftige ökotoxikologische Bewertungen von belasteten Sedimenten bieten.

\section{Literatur}

Austen MC, McEvoy AJ (1997) The use of offshore meiobenthic communities in laboratory microcosm experiments: Response to heavy metal contamination. J Exp Mar Biol Ecol 211:247-261

Austen MC, Somerfield PJ (1997) A community level sediment bioassay applied to an estuarine heavy metal gradient. Mar Environ Res 43:315-328

Beyrem H, Mahmoudi E, Essid N, Hedfi A, Boufahja F, Aissa P (2007) Individual and combined effects of cadmium and diesel on a nematode community in a laboratory microcosm experiment. Ecotoxicol Environ Saf 68:412-418

Bongers T, Ferris H (1999) Nematode community structure as a bioindicator in environmental monitoring. Trends Ecol Evol 14:224-228

Bongers T, Ilieva-Makulec K, Ekschmitt K (2001) Acute sensitivity of nematode taxa to $\mathrm{CuSO}_{4}$ and relationships with feeding-type and life-history classification. Environ Toxicol Chem 20:1511-1516

Brack W (2003) Effect-directed analysis: a promising tool for the identification of organic toxicants in complex mixtures? Anal Bioanal Chem 377:397-407
Bryan GW, Langston WJ (1992) Bioavailability, accumulation and effects of heavy-metals in sediments with special reference to United-Kingdom estuaries - a review. Environ Pollut 76:89-131

Chapman PM, Hollert H (2006) Should the sediment quality triad become a tetrad, a pentad, or possibly even a hexad? J Soils Sediments 6:4-8

Coull BC, Chandler GT (1992) Pollution and meiofauna - field, laboratory, and mesocosm studies. Oceanogr Mar Biol 30:191-271

Garric J, Vollat B, Duis K, Pery A, Junker T, Ramil M, Fink G, Ternes TA (2007) Effects of the parasiticide ivermectin on the cladoceran Daphnia magna and the green alga Pseudokirchneriella subcapitata. Chemosphere 69:903-910

Giere O (2009) Freshwater Biotopes. In: Giere O (ed) Meiobenthology. The Microscopic Motile Fauna of Aquatic Sediments. Springer, Berlin Heidelberg, pp 328-348

Gyedu-Ababio TK, Baird D (2006) Response of meiofauna and nematode communities to increased levels of contaminants in a laboratory microcosm experiment. Ecotoxicol Environ Saf 63:443-450

Halley BA, Jacob TA, Lu AYH (1989) The environmental-impact of the use of Ivermectin - environmental-effects and fate. Chemosphere 18:1543-1563

Heininger P, Höss S, Claus E, Pelzer J, Traunspurger W (2007) Nematode communities in contaminated river sediments. Environ Pollut 146:64-76

Höss S, Traunspurger W, Severin GE, Juttner I, Pfister G, Schramm KW (2004) Influence of 4-nonylphenol on the structure of nematode communities in freshwater microcosms. Environ Toxicol Chem 23:1268-1275

Kennedy AD, Jacoby CA (1999) Biological indicators of marine environmental health: Meiofauna - A neglected benthic component? Environ Monit Assess 54:47-68

Korthals GW, van de Ende A, van Megen H, Lexmond TM, Kammenga JE, Bongers T (1996) Short-term effects of cadmium, copper, nickel and zinc on soil nematodes from different feeding and lifehistory strategy groups. Appl Soil Ecol 4:107-117

Long ER, Chapman PM (1985) A sediment quality triad - measures of sediment contamination, toxicity and infaunal community composition in Puget-Sound. Mar Pollut Bull 16:405-415

Millward RN, Carman KR, Fleeger JW, Gambrell RP, Powell RT, Rouse MAM (2001) Linking ecological impact to metal concentrations and speciation: A microcosm experiment using a salt marsh meiofaunal community. Environ Toxicol Chem 20:2029-2037

Schratzberger M, Wall CM, Reynolds WJ, Reed J, Waldock MJ (2002) Effects of paint-derived tributyltin on structure of estuarine nematode assemblages in experimental microcosms. J Exp Mar Biol Ecol 272:217-235

Smit CE, Schouten AJ, Van den Brink PJ, van Esbroek MLP, Posthuma L (2002) Effects of zinc contamination on a natural nematode community in outdoor soil mesocosms. Arch Environ Con Tox 42:205-216

Suderman K, Thistle D (2003) A microcosm system for the study of pollution effects in shallow, sandy, subtidal communities. Environ Toxicol Chem 22:1093-1099 